\title{
Microbiology Susceptibility Domain
}

National Cancer Institute

\section{Source}

National Cancer Institute. Microbiology Susceptibility Domain. NCI Thesaurus. Code C61531.

A subject domain utilized for the submission of information encompassing and representing data, vocabulary or records related to microbiology susceptibility tests. 